www.nature.com/jea

\title{
EDITORIAL
}

\section{Exposure assessment: utility and application within homeland or public security}

Journal of Exposure Analysis and Environmental Epidemiology (2004) 14, 427-428. doi:10.1038/sj.jea.7500376

Prior to the terrorist attack on the World Trade Center and the anthrax letters in 2001, the scientific and social issues confronting the field of Environmental Health Sciences focused primarily on examining and reducing long-term low-level exposures and potential health outcomes to environmental toxicants. Issues like developmental effects in children, environmental cancers, asthma, transitory effects from acute exposure to ozone and secondary particles, and life-shortening effects of $\mathrm{PM}_{2.5}$ were major concerns in the United States and Western Europe. This is in contrast to previous concerns about more easily identifiable effects, for example, episodic increases in death, caused by the Londontype smog prior to the 1960s from coal used for space heating and industrial sources. Such situations still exist in developing countries and are beginning to be addressed by various organizations.

In the background, however, the military and others (e.g., National Laboratories) have maintained vigilance about the debilitating acute effects and death that can result from shortterm exposures to biological, physical and chemical toxicants that are referred to as Warfare Agents. Further, the USEPA commissioned a National Research Council committee to develop Acute Exposure Guidelines for Community Settings (AEGLs) for some of these toxicants. Up until 9-11-01, these efforts received little attention. In fact, I had not seriously considered the use of chemical warfare agents, etc. against US citizens on American soil. It was a distant thought, an event that could only happen to others, for example, the Kurds in Iraq. How naïve!

Emergency response to acute toxicant exposures has always been a part of catastrophe planning. However, the approaches rarely considered the consequences of deliberate terrorist acts designed to kill and maim the general public, especially within America. The mainstream exposure community also has not focused attention on chemical/biological or radiological weapons of war. However, the attack on the WTC, and the anthrax letters made it clear that we were thrust into a different world. As a number of people have said, our lives have been changed for the seeable future or forever.

The "new" era of Homeland or Public Security is beginning to make demands on the fields that comprise environmental health sciences including exposure analysis.
I recently attended international conferences/workshops on the topic and it is clear that the dynamics for interaction and planning between diverse disciplines is beginning to evolve. However, there needs to be a health-based framework to respond effectively to a low probability event that leads to significant acute exposures and deadly or severely debilitating health outcomes. Unfortunately, our field has already been placed in a weakened position because of the 2003 NRC Report "Tracking and Predicting the Atmospheric Dispersion of Hazardous Material Releases: Implications for Homeland Security." It recommended three major features of a response program, but it was programmatically insufficient. Their recommendations placed high priority on (1) dispersion modeling, (2) measurement of contaminants (sensors), and (3) joint efforts with emergency responders. Missing were exposure and dose assessments to identify populations at risk, due to primary and/or secondary contact, and how to adjust individual/population postevent activities and behavior to reduce casualties. In addition, Centers currently planned by the Homeland Security Department do not focus specifically on exposure issues.

Areas of exposure research that can assist in addressing homeland or public security issues are: (1) response monitoring, (2) response planning and training, (3) scenario development, (4) characterization of individual and population activities, (5) approaches for exposure reduction during/ after an event, (6) acute risk assessment during and postevent, and (7) clean-up and re-entry criteria. Prevention, a primary goal, must be left to the FBI, CIA, DHS, and other law enforcement agencies.

The opportunities for our field begin with the application of the source-to-dose-effects continuum, and development and evaluation of release scenarios. Such results can be employed to assess the potential magnitude of population impact and to determine ways to reduce the magnitude and the extent of exposures and health outcomes through appropriate desktop and field exercises. For deployed troops, the locations and methods of release are more predictable than the general population since contact will be related to military patterns of activity. Thus, the types of exposure analyses that we currently conduct, can be used prospectively to reduce "uncertainty" about the types and intensity of exposure that can be expected within the general public for an 
emergency. Beyond the major issue of activity patterns, other difficult tasks for scenario development include: (1) biological, physical or chemical agent and (2) method of delivery.

Analyses would estimate the number and distribution of casualties, the potential zone and duration of infection or debilitating effects, and the range in magnitude and extent of contamination, a point not developed or acknowledged in the NRC report. The results can define criteria and activities for the location of postevent monitoring systems, media-specific transport and dilution patterns, selection of protective gear, the degree of chaos, exposure reduction strategies (e.g. remaining indoors, evacuation), post-event monitoring strategies, for example, portability, response time for deployment, pollutant selection, and location, and logistics. In contrast to other environmental issues, we have had little experience in dealing directly with the use of chemical/ biological/radiological weapons (CBR) on the general public, and there are few well-defined modeling and measurement protocols, and strategies for rapid response. Although air is an obvious concern, scenarios must include the possibility for attacks on water and food supplies. From the standpoint of the indoor environment, the source can yield exposures via multiple pathways.

Among the benefits of applying the principles of exposure analysis and assessment to homeland security issues will be semi-realistic evaluation of the timing of an event and the characterization of the speed with which decisions must be made to minimize the total impact on human health. We can also provide perspective on the level of acute health effects to be experienced by victims of a highly toxic exposure, on the methods needed for better risk communication. This can assist in minimizing the risk to the general public by modification of behavior during and after an attack, and in mitigating the severity of the impact of an attack.

Paul J. Lioy, Ph.D. Professor and Deputy Director for Governmental Relations, Environmental and Occupational Health Sciences Institute, UMDNJ/RWJMS and Rutgers University 